\title{
Hodgkin lymphoma misdiagnosed as Kikuchi-Fujimoto disease - how important it is to confirm an unusual diagnosis
}

\author{
Authors: Wiktoria Grycuk ${ }^{1}$, Piotr Krych ${ }^{2}$ (mentor) \\ ${ }^{1}$ Faculty of Medicine, Medical University of Warsaw, Poland \\ ${ }^{2}$ Oncology Department, The Children's Memorial Health Institute, Warsaw, Poland
}

DOI: https://doi.org/10.26800/LV-142-supp5-7

\section{Background:}

Kikuchi-Fujimoto disease (KFD) is an extremely rare, self-limiting disorder of unknown etiology, with most cases occurring in Asia. The treatment of KFD is mainly supportive with NSAIDs or steroids in isolated cases. Its clinical presentation and histopathological findings may overlap with more common disorders, including malignancies such as Hodgkin Lymphoma (HL). It's essential to thoroughly reevaluate such diagnoses before initiating the treatment.

\section{Case presentation:}

A 6-year-old boy was admitted to the regional hospital's pediatric ward with a high fever, cervical lymphadenopathy, enlarged liver and spleen. Before admission, he was treated with antibiotics (clarithromycin and clindamycin) with no response. After exclusion of infectious origin in laboratory tests, the imaging studies and biopsies of bone marrow and cervical lymph nodes were performed. Based on the histopathological examination, the diagnosis of Kikuchi-Fujimoto disease was established. There were no further consultations prior to treatment with methylprednisolone. Three months later, on admission to Pediatric Oncology Department, the patient presented with generalized lymphadenopathy, jaundice, hepatosplenomegaly and ascites. Laboratory tests showed anemia, thrombocytopenia, hyperbilirubinemia, elevation of inflammatory markers and features of liver insufficiency. The histopathological specimens were reviewed in a reference center where diagnosis of Anaplastic Large Cell Lymphoma (ALCL) and HL was considered. The chemotherapy regimen for ALCL was administered. Soon after, the definitive diagnosis of HL was confirmed. Chemotherapy was modified resulting in significant clinical improvement.

\section{Conclusion:}

Every extremely rare diagnosis should be carefully reconsidered and confirmed in a reference center. Our case shows that misdiagnosis that entails inappropriate treatment may result in worsening of prognosis and life-threatening compilations for the patient.

Keywords: Hodgkin Lymphoma; Kikuchi-Fujimoto disease; misdiagnosis 